\title{
Cerebellar Fastigial Nucleus Electrical Stimulation Alleviates Depressive-Like Behaviors in Post-Stroke Depression Rat Model and Potential Mechanisms
}

\author{
Lei Zhang ${ }^{\text {a }}$ Mingkui Zhao ${ }^{\text {bu-Bo Suib }}$ \\ aSchool of Nursing, Jinzhou Medical University, Jinzhou, Liaoning, bDepartment of Neurology, First \\ Affiliated Hospital of Jinzhou Medical University, Jinzhou, Liaoning, China
}

\section{Key Words}

Cerebellar FNS • PSD • rCBF • Cerebellar Purkinje cells • Cytokines

\begin{abstract}
Objective: To identify the molecular mechanism of post-stroke depression (PSD), and observe the therapeutic effects of cerebellar fastigial nucleus electrical stimulation (FNS) on the behaviors and regional cerebral blood flow (rCBF) in a PSD rat model. Methods: Healthy SD rats were randomly divided into four groups (sham, stroke, post-stroke depress and FNS group). Sham group $(n=6)$ underwent sham operation. The other three groups $(n=6 * 3)$ underwent MCAO. Rats were examined twice a week in open filed test. Moreover, neuroprotective effect on cerebellar Purkinje cells and expression of cytokines in hippocampal tissue were examined. Results: The PSD group showed a significant weight loss, decreased consumption of sucrose water, reduced rearing and locomotor activities. The FNS significantly alleviates the body weight loss and sucrose preference, locomotor and rearing activities. The bilateral rCBF was also restored after FNS treatment. Moreover, FNS improved the neuroprotection via suppressing apoptosis of cerebellar Purkinje cells. And the inflammatory cytokines mRNA level in hippocampus was significantly decreased. Conclusion: FNS treatment alleviates depressive-like behaviors and rCBF in PSD rats model, which could be attributed to its ability to protect cerebellar Purkinje cells and decrease the mRNA level of inflammatory cytokines.
\end{abstract}

\section{Introduction}

One of the most frequent psychiatric complications of stroke is post-stroke depression (PSD). Its incidence is about $33-40 \%$ in stroke patients and approximately 3 million Americans are affected each year [1,2]. Stroke survivors may have difficulty in identifying

Professor Ru-Bo Sui

KARGER
Department of Neurology, First Affiliated Hospital of Jinzhou Medical University No.2 Renmin Rd, Jinzhou, Liaoning, 121000 (China)

E-Mail profproton@163.com 


\section{Cellular Physiology Cell Physiol Biochem 2017;41:1403-1412 \\ and Biochemistry Published onIIne: IVarch 16, $2017 \quad$\begin{tabular}{l|l} 
DOI: 10.1159/000467940 2017 The Author(s). Published by S. Karger AG, Basel \\
www.karger.com/cpb
\end{tabular} \\ Zhang et al.: Cerebellar FNS Alleviates Depressive-Like Behaviors in PSD Rat Model}

and explaining symptoms of depression, especially older stroke survivors with higher morbidities. Stroke patients with PSD are at higher risk of death and achieve a mild improvement in rehabilitation programs compared with stroke patients without depression. As a special psychiatric disorder and most common complication following stroke, PSD is usually tricky to treat [2].

Gliogenesis and neurogenesis are formed as an integral part of the adaptive reaction of the brain responding to ischemia. Likely, it has been reported that hippocampal neurogenesis is enhanced by transient global ischemia in rats [3, 4]. Ischemia adequately enhances proliferation of resident glial cells in majority of non-neurogenic regions of the adult brain [5,6]. In a recent study, unexpected chronic minor stress following left MCAO has been shown to cause depressive-like behaviors as well as reduced degrees of hippocampal neurogenesis in rats $[7,8]$. Moreover, anti-depressant therapy with citalopram reversed both the antineurogenic and the behavioral impacts of persistent stress following brain ischemia [8]. Recently, it was reported that the cerebellum may exhibit a role in psychiatric illness, behavior and cognition $[9,10]$. Cerebellum dysfunction might be involved in pathogenesis of PSD, based on the correlation between the cerebellum and the affective disorder, similarity in pathology between PSD and neuropsychiatric diseases (particularly for depression), and the clinical trial indicating that cerebella substantially relives symptom of PSD upon activation [10]. But there is little evidence that investigates the putative cerebellar effect in PSD [11].

Several clinical studies suggested that symptom of PSD can be substantially eased by activated cerebella. Sui et al. discovered that emotion disorders in post-stroke patient can be improved by fastigial nucleus electrical stimulations [12]. Likely, Su et al. reported that electrical stimulation cerebellar fastigial nucleus showed substantially alleviated symptom of patients who had PSD, in comparison to healthy controls [13]. Electrical stimulation excites fibers passing through the fastigial nucleus, leading to increased blood pressure, reflexive vascular expansion, and increased rCBF [14]. FNS can pass fibers exceeds the sympathetic inhibitory response by FN nerve cells. FNS can significantly enhance the tolerance of brain tissue to subsequent cerebral ischemia. Robust data show that electrical stimulation of the cerebellar fastigial nucleus can elicit marked global protection against brain injury in rats [15-17]. Actually FNS as a therapeutic choice is available in our hospital to treat some mental illnesses including PSD.

Many studies have sought to explore the mechanisms that might account for the neuroprotection elicited by FNS. We focus on those mechanisms and the clinical applications of FNS in treatment of PSD. Postmortem brains of patients with depression exhibit neuronal apoptosis and DNA fragmentation, indicating an increased neuronal vulnerability in patients with depression [18]. In animal models of mood disorders, reduced neurogenesis and neuronal loss have also been found [18-20]. Two mechanisms may involve in neuronal cell death, the delayed form, apoptosis, or an acute form, or necrosis that happens rapidly [21].

In this study, we showed that the FNS treatment significantly alleviated the body weight loss and depressive-like behaviors. Further studies showed that the rCBF were significantly increased by the FNS, compared with the PSD rats. The neuroprotection of cerebellar Purkinje cells mediated by FNS were confirmed with electronic microscope and immunostaining of activated caspas-3.

\section{Materials and Methods}

\section{Establishment of PSD rat model}

The Sprague-Dawley rats were obtained from Animal Center, Liaoning Medical College and maintained in plastic cages. The conditions were controlled at room temperature and relative humidity of 50-60\%. The rats were fed with tap water and standard rat diet. Twenty four healthy Sprague Dawley rats with uniform behavior were selected and randomly divided into four groups as follows: Sham, stroke, PSD and FNS groups. In the sham group, sham operated rats underwent the same surgical procedure, except the suture was not introduced into the artery. The rats underwent MCAO and divided into 3 groups: stroke, 


\section{Cellular Physiology Cell Physiol Biochem 2017;41:1403-1412 \\ \begin{tabular}{l|l} 
and Biochemistry Published onIne: VIarch 16, 2017 & $\begin{array}{l}\text { DO } 2017 \text { The Author(s). Published by S. Karger AG, Basel } \\
\text { www.karger.com/cpb }\end{array}$
\end{tabular} \\ Zhang et al.: Cerebellar FNS Alleviates Depressive-Like Behaviors in PSD Rat Model}

PSD and FNS group. The PSD and FNS rats were subjected to isolation-housing in combination with chronic unexpected mild stress (CUMS, water deprivation, wet litter, fasting, behavioral restriction, tail clamping, electric shock to foot and ice-water swimming) to set up a depression model. The study protocol was approved by the research ethnic committee at Liaoning Medical College.

\section{Cerebellar fastigial nucleus electrical stimulation}

Rats were fasted for 8-12 hours, 3.5\% (w/v) chloral hydrate $(10 \mathrm{~mL} / \mathrm{kg}$, intraperitoneally) was used to anesthetize the rats, and a brain stereotactic endoscope (Japan Mau Science Equipment Research Institute, Tokyo, Japan) was used to immobilize the rats in accordance with the manufacturer's protocol. Stereotaxic atlas of the rat brain were used to accurately positioned the fastigial nucleus, and set the posterior border of the anterior fontanelle as the zero point, an 11.1-mm incision performed, YC-2 programmed electrical stimulation instrument (Chengdu Instrument Factory, Chengdu, Sichuan Province, China) with a 70- $\mu \mathrm{A}$ direct-current square-wave pulse $(50 \mathrm{~Hz})$ was used to perform the attachment of electrodes in a hole made in the skull. All the tests were performed three times. The Rat body weights, consumption of sucrose water, rearing activity, locomotor activity, $\mathrm{rCBF}$ of all the rats were collected during the research.

\section{Locomotor activity}

The general locomotor activity were performed on the OFT (open field test). Rats were placed in the front right corner of a clear acrylic box (16" × 16") for $20 \mathrm{~min}$. A computer operated PAS Open Field system (San Diego Instruments, San Diego, CA) was used to quantify the locomotor activity as the total number of beam breaks in accordance with the manufacturer's instruction.

\section{Regional cerebral blood flow (rCBF)}

A $2 \mathrm{MHz}$ pulsed transcranial Doppler ultrasound (TCD) system (Spencer Technologies, Seattle, WA, USA) was used to measure the rCBF in the middle (MCAv) (Contra) and posterior (PCAv) (Contra) cerebral arteries continuously throughout each experiment in accordance with the manufacturer's instruction. The standardized procedures were used to determine the location and identification of MCA (Contra) and PCA (Contra) as described by Willie et al. [22]. A headband fixation device (Mark600, Spencer Technologies, Seattle, WA, USA) was used to fix and hold the Bilateral TCD probes

\section{Electron microscopy}

Rats were euthanized and the sections on adjacent grids during photography. The sections were stained with uranyl acetate and lead citrate, and were analyzed using a Philips TEM/CM 10 electron microscope.

\section{RNA isolation and real-time PCR}

TRIzol reagent (Invitrogen, California, USA) was used to extract the total RNA from tissue samples according to the manufacturer's protocol. Real-time quantitative PCR (qPCR) was used to evaluate the mRNA level of TNF- $\alpha$, IL-6, and IL-1 $\beta$ mRNAs levels. Superscript II Reverse Transcriptase (Invitrogen, CA, USA) was used to reverse transcribes the RNA isolated to cDNA with an oligo dT18 primer in accordance with the manufacturer's instruction. The Sequence Detection System 7900HT (Applied Biosystems, Foster City, CA) containing the Universal Master Mix (PE Applied Biosystems, Foster City, CA) was used to perform the teal-time PCR with specific primers in order to quantify the mRNA levels. All tests were performed at least three times. The GAPDH was used as an internal control for normalization of TNF- $\alpha$, IL- 6 , and IL-1 $\beta$ mRNAs expression. The $\Delta \Delta$ Ct method was used to calculate the expression of the cytokines. All tests were performed at least three times.

\section{Immunohistochemistry}

Immunohistochemistry was performed on brain sections using a rabbit polyclonal antibody directed against Cleaved caspase-3 (cell signaling technology, \#9664). Animals were perfused using 4\% paraformaldehyde in PBS. Brains were post-fixed in the same fixative overnight at $4^{\circ} \mathrm{C}$ and cryoprotected overnight at $4^{\circ} \mathrm{C}$ in PBS containing 30\% sucrose. Ten micrometer parasagittal sections were cut using a cryostat and incubated overnight at $4^{\circ} \mathrm{C}$ using Cleaved caspase-3 antibody (dilution 1:1000). Immunocomplexes were then revealed using HRP (horseradish peroxidase)-conjugated secondary antibody was used to treat the sections at room temperature for $30 \mathrm{~min}$. 3, 3'-diaminobenzidine tetrahydrochloride solution was used to treat the sections, Hematoxylin and eosin (H\&E) stains was used to counterstain all the sections. 


\section{Cellular Physiology Cell Physiol Biochem 2017;41:1403-1412 \\ \begin{tabular}{l|l|l} 
and Biochemistry Publisned onIIne: IVIarch 16, 2017 & $\begin{array}{l}\text { (c) } 2017 \text { The Author(s). Published by S. Karger AG, Basel } \\
\text { www.karger.com/cpb }\end{array}$
\end{tabular}

\section{Statistical analysis}

All data shown here were in the form of mean \pm SD (standard deviation) unless specially specified. SPSS15.0 software (SPSS Inc., Chicago, IL, USA) was used to perform the statistical analysis, the comparisons between two groups were carried out using Student's t-test, and comparisons among three groups were done using ANOVA, occurrence analysis were performed using Chi-squared test. A p-value less than 0.05 were considered significant statistically.

\section{Results}

Establishment of the post-stoke depression rat model

Healthy Sprague Dawley rats were subjected to MACO. The PSD and FNS rats were further in combination CUMS to develop depress rats model. The FNS group were treated with FNS as described in the methods section. One week after the initiation of the treatment, more symptoms including slow response, drowsiness and sluggishness were recorded without any unexpected death.

\section{Cerebellar FNS alleviates depressive-like behaviors}

In our post-stoke depressive-like rat model, PSD group show depressive-like behaviors, such as body weight-loss (Fig. 1), sucrose preference (Fig. 2), locomotor activities (Fig. 3) and rearing activities (Fig. 4) in OFT. After FNS treatments, the rats significantly gain body weight (since day $21^{\text {st }}$ ) and sucrose preference (since day $17^{\text {th }}$ ), locomotor activities and rearing activities in open filed test (since Day $21^{\text {st }}$ ). These data indicate that cerebellar FNS significantly alleviates depressive-like behaviors in the post stoke depressive-like rat models.

Fig. 1. Dynamic changes in rat body weight. Rat body weights in the stroke and FNS groups were reduced before Day 11, and subsequently the body weights increased. The body weights in the PSD group continually decreased and reached their lowest level on Day 30. $\nabla P<0.01$, vs. stroke group; $\star P<0.05$, vs. PSD group. The bars represent $\mathrm{SD}$. $\mathrm{N}=6$.

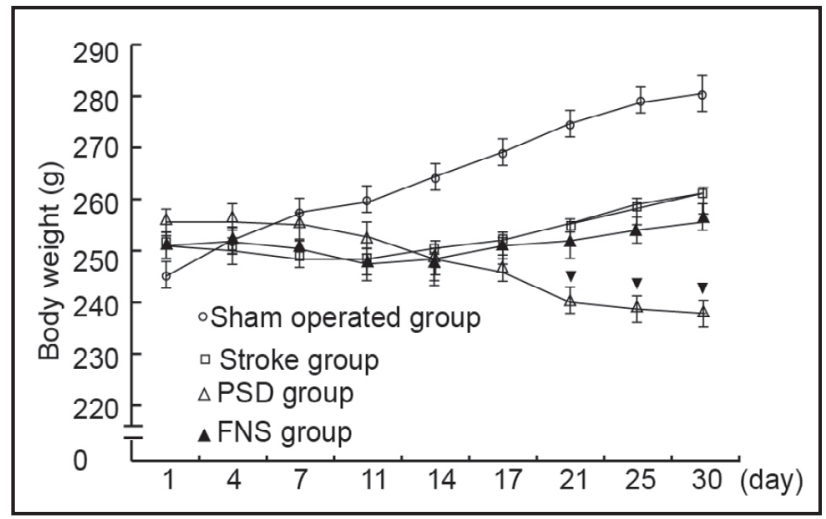

Fig. 2. Dynamic changes of sucrose preference. The consumption of sucrose water decreased in the PSD and the FNS groups during the first 11 days compared with sham-operated and stroke groups. Subsequently, the consumption in the FNS group increased slightly from Day 14 and remained constant thereafter. The consumption of sucrose water in the PSD group continually decreased and reached the lowest level on Day 25. $\nabla P<0.01$, vs. stroke group; $\star P<$ 0.01 , vs. PSD group. The bars represent SD. $\mathrm{N}=6$.

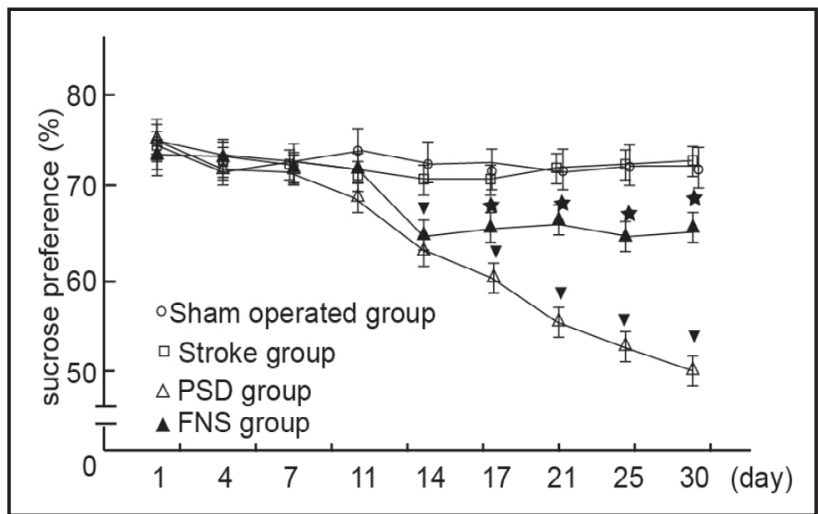


Fig. 3. Dynamic changes of locomotor activity in the open-field test. The scores for locomotor activity in the sham-operated and stroke the groups were not significantly different. Locomotor activity in the PSD group was significantly reduced compared with the stroke group and reached its lowest point on Day 30. Locomotor activity in the FNS group decreased up till Day 17 and then it increased slightly. The difference between the PSD and FNS groups was statistically significant after Day 21. $\nabla P<0.01$, vs. stroke group; $\star P<0.01$, vs. PSD group. The bars represent SD. $\mathrm{N}=6$.

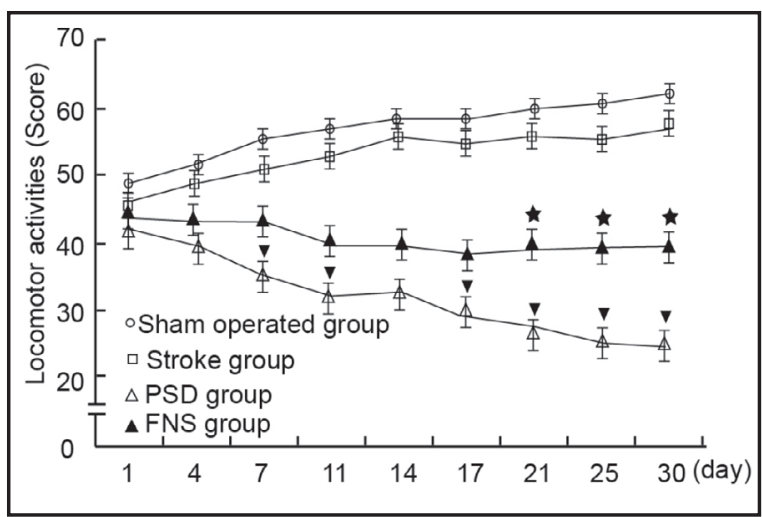

Fig. 4. Dynamic changes of rearing activity in the open-field test. The scores for rearing activity in the sham-operated and stroke groups were not significantly different. Rearing activity in the PSD group was significantly reduced compared with the stroke group and reached its lowest point on Day 30. Rearing activity in the FNS group decreased up till Day 17 and then it increased slightly. The difference between the PSD and FNS groups was statistically significant after Day 21. $\nabla P<$ 0.01 , vs. stroke group; $\star P<0.01$, vs. PSD group. The bars represent SD.

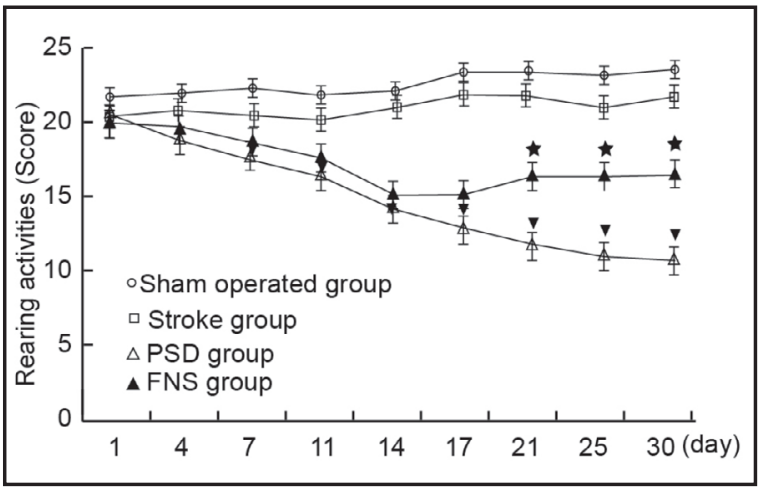

Fig. 5. Dynamic changes of bilateral rCBF. The cerebellar rCBF (contra) in the stroke group was reduced on Day $1(P<0.05)$ compared with the sham-operated group, and then it increased and recovered after Day 17. The cerebellar rCBF (contra) in the PSD group significantly decreased compared with the stroke group until the lowest point was reached on Day 25. For the FNS group, cerebellar rCBF (contra) had a similar rCBF profile as that of the stroke group except it had a relatively higher $\mathrm{rCBF}$. $P<0.05$, vs. sham-operated group; $\boldsymbol{\nabla} P<0.01$, vs. stroke group; $\star P<0.01$, vs. PSD group. The bars represent SD.

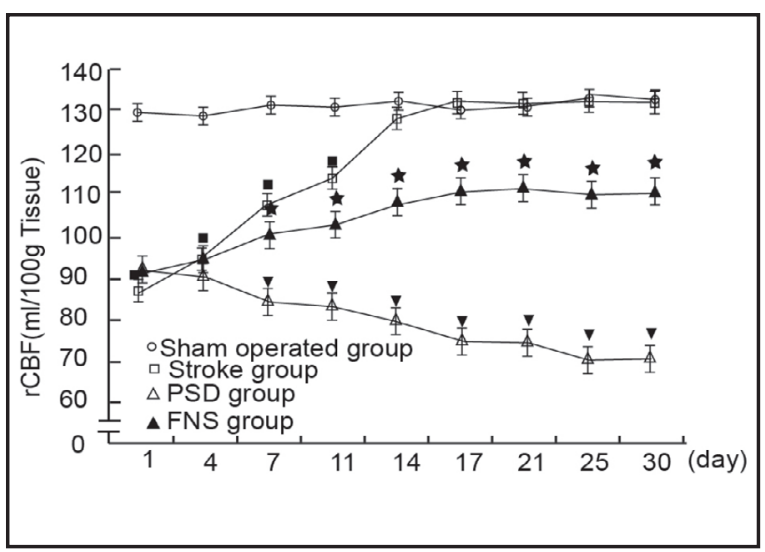

\section{Cerebellar FNS significantly restore bilateral $r C B F$}

The enhancement of $\mathrm{rCBF}$ is tightly and positively coupled to increases in synaptic glucose metabolism and electrical activity $[23,24]$. We also examine if Cerebellar FNS affects the rCBF in the PSD rat model. our data show that the rats in PSD group was significantly lost their $\mathrm{rCBF}$ since day $7^{\text {th }}$; moreover, the FNS treatments could significantly increase the rCBF in since day $5^{\text {th }}$ (Fig. 5).

\section{Cerebellar FNS protects cerebellar Purkinje cells}

Cerebellar Purkinje cells are well-known for synaptic plasticity. Here, we investigated the ultrastructure of the cerebellar Purkinje cell terminals in the cerebellar nuclei as well as the activities of their target neurons. In the sham and stoke groups ultrastructure of the Purkinje cells (contra) in the stroke rats appeared slightly damaged (Fig.6 B) comparing with Sham rats (Fig. 6A). The ultrastructure of Purkinje cells in PSD rats showed clear signs 


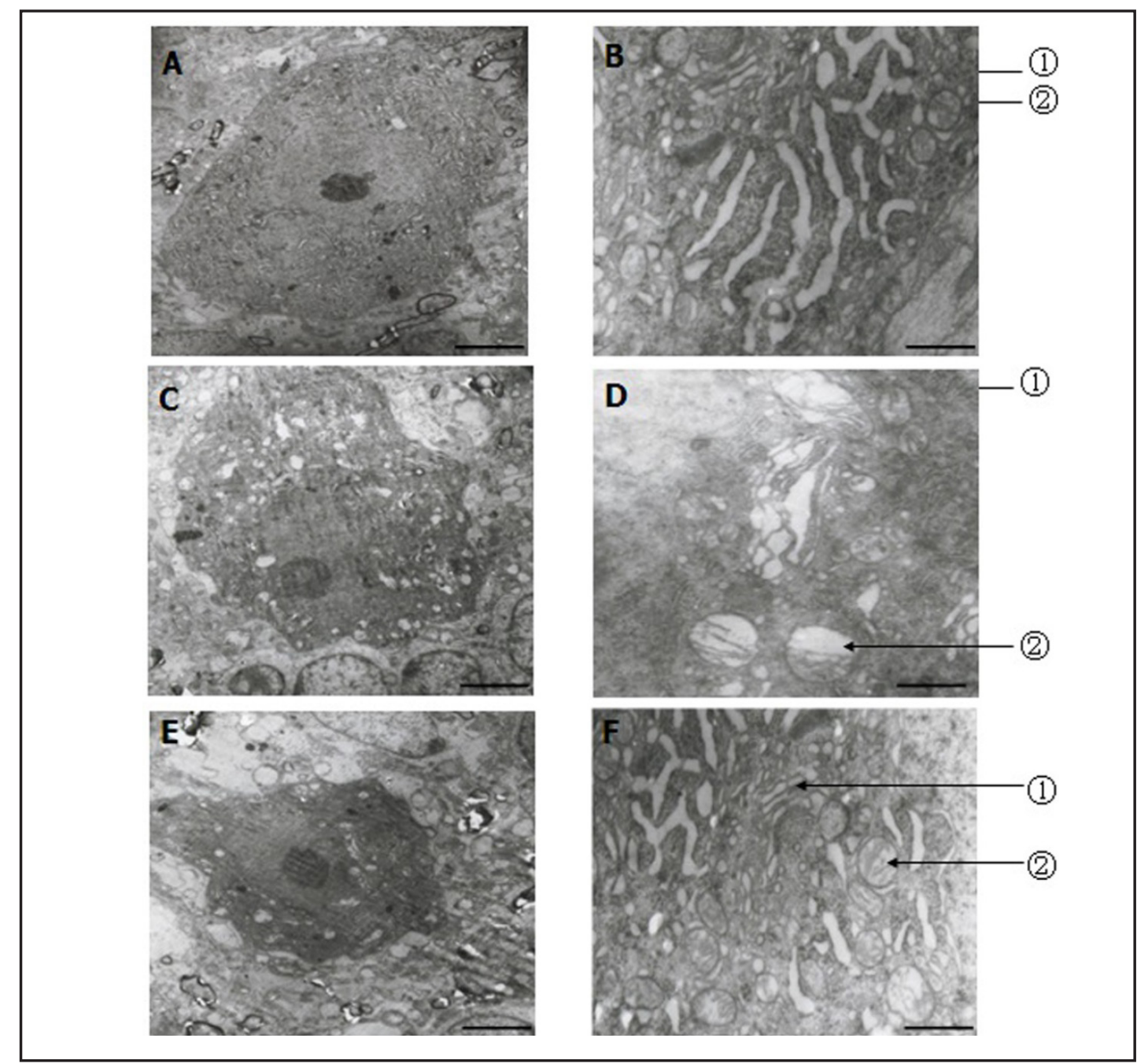

Fig. 6. Neuroprotection of FNS on cerebellar Purkinje cells on Day 14. The ultrastructure of Purkinje cells (ipsi) in all groups remained mostly unchanged. The ultrastructure of the Purkinje cells (contra) in the stroke rats appeared slightly damaged (A, B). Magnification $\times 4000$ for A and $\times 14000$ for $B$. The ultrastructure of Purkinje cells in PSD rats showed clear signs of damage (C, D). Magnification $\times 3500$ for $C$ and $\times 12000$ for D. Evidence of cellular damage was reduced in the FNS group (E, F) Magnification $\times 3500$ in E, and $\times 12000$ in F .(1) = Expanded endoplasmic reticulum, (2) = vague and swollen mitochondria. The images on the right are magnifications of the images on the left. Scale bars: $4 \mu \mathrm{m}$ in $\mathrm{A}, \mathrm{C}$, and $\mathrm{E} ; 1 \mu \mathrm{m}$ in $\mathrm{B}, \mathrm{D}$, and F. N=3.

of damage (Fig. 6, C \& D). Meanwhile, the cellular damage was reduced in the FNS group (Fig. $6, \mathrm{E} \& \mathrm{~F})$.

In addition, we examined the pro-apoptosis marker, activated caspas-3, in the cerebellar Purkinje cells. Comparing with sham-operated rats (Fig. 7A), the immunostaining of activated caspase-3 is significantly higher in stroke group (Fig. 7B), and even higher in the PSD rats (Fig. 7C). The cerebellar FNS will dramatically decrease the immunostaining of caspase-3 (Fig. 7D), which indicated that the FNS protects the cerebellar Purkinje cells from apoptosis induced by PSD.

Cerebellar FNS decrease expression of inflammatory cytokines

Significantly higher levels of inflammatory markers are associated with a range of depressive symptoms. Here, we examine the mRNA level of the TNF- $\alpha$, IL- 6 , and IL- $1 \beta$ in hippocampal tissue (Fig. 8, A-C, respectively). The stroke rats have a transient cytokines 
Fig. 7. Effects of FNS on caspase-3 activation in cerebellar Purkinje cells. There were very few Purkinje cells that were immunostained for activated caspase- 3 in the sham-operated group (A). The level of activated caspase- 3 significantly increased $(P<0.001)$ in the stroke group (B) compared with the sham-operated group. Compared with stroke group, the level of activated caspase- 3 in the PSD group (C) was significantly higher $(P<0.001)$. The level of activated caspase-3 in the FNS group (D) was significantly reduced $(P<0.001)$ compared with the PSD. $\mathrm{N}=3$.
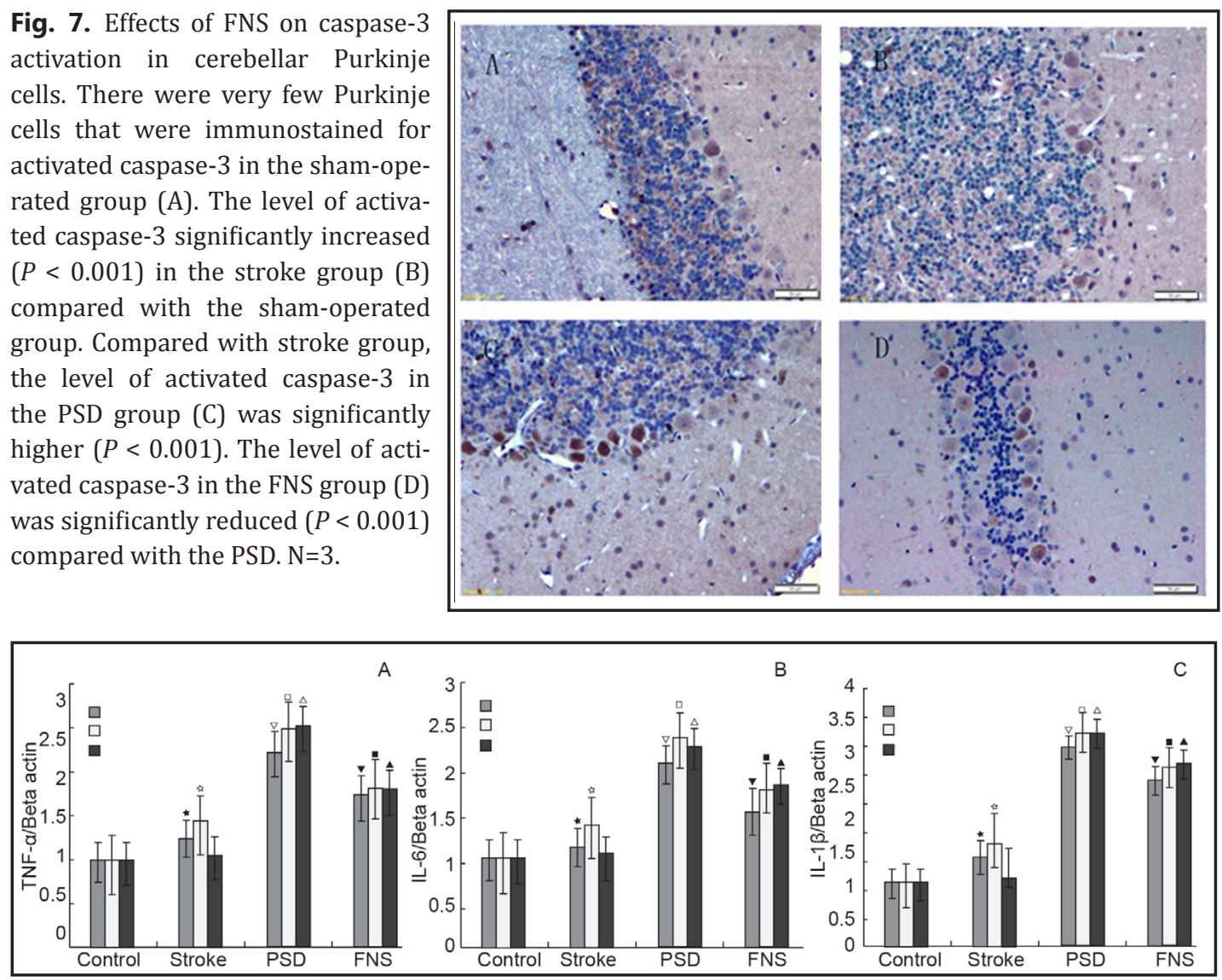

Fig. 8. Effects of FNS on expression of TNF- $\alpha$, IL-6, and IL-1 $\beta$ in hippocampal tissue. The expressions of TNF- $\alpha$, IL-6, and IL-1 $\beta$ mRNAs (A-C, respectively) in hippocampal tissue of PSD rats on Days 7, 14, and 21. Compared with the sham-operated group, the levels of cytokine mRNAs in the stroke group increased within 14 days, and then the levels decreased to their lowest levels on Day 21. Compared with the stroke group, the levels of the cytokine mRNAs in the PSD group significantly increased within 14 days, and then they remained mostly stable after Day 14. In the FNS group, mRNA levels displayed a similar profile as the PSD group, but the mRNA levels were significantly lower than the PSD group. $\star P<0.05$, vs. control group

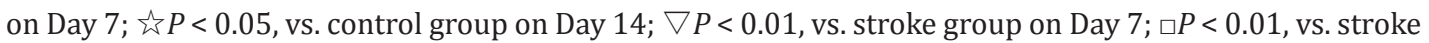
group on Day 14; $\triangle P<0.01$, vs. stroke group on Day $21 ; \boldsymbol{\nabla} P<0.05$, vs. PSD group on Day $7 ; \boldsymbol{\square} P<0.05$, vs. PSD group on Day 14; $\Delta \mathrm{P}<0.05$, vs. PSD group on Day 21. The bars represent SD. $\mathrm{N}=3$.

expression upregulation on day $7^{\text {th }}$ and $14^{\text {th }}$, comparing with sham rats. The PSD rats have significantly upregulated expression of cytokine since day $7^{\text {th }}$ to the day $21^{\text {st }}$. The PSD rats with FNS have significantly lower expression level of cytokines, which is consistence with the decrease of the depressive symptoms in the FNA rats.

\section{Discussion}

Post-stroke depression (PSD) is highly clinical relevance event. At least $1 / 3$ of survivors from stroke present with mood symptoms sometimes following the event $[1,25$, 26]. Depression following stroke often goes through a chronic phase and is correlated with diverse poor health outcome such as morbidity, mortality and aggravated disability [27-30]. Intriguingly, during the chronic phase following stroke, symptoms of depression may even aggravate [2]. Older patients with acute stroke experience depression after 20 months have poorer physical and cognitive outcome [31]. 


\section{Cellular Physiology Cell Physiol Biochem 2017;41:1403-1412

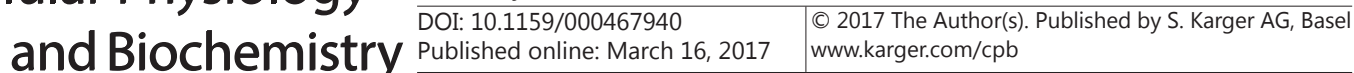 \\ Zhang et al.: Cerebellar FNS Alleviates Depressive-Like Behaviors in PSD Rat Model}

FNS renders the brain to prevent neuronal damage for up to 2 weeks following stimulation [15]. This inhibition of apoptosis provided by FNS was modest, being decreased by about $30 \%$ when compared to controls, and relatively lower than expected from in vivo studies in which there are more than 50\% maximal decreases in cell death [15-17]. This may reveal the property of the in vitro system in that adult neurons are vulnerable in culture, so the complete protective effect of FNS is not fully reflected in this system. Movever, in vivo neuroprotection by FNS may be associated with several protective mechanisms in addition to anti-apoptosis, including the systemic inhibition of inflammatory responses [32]. For instance, interleukins 1 and 6 (IL-1, IL-6) and tumor necrosis factor (TNF) had already been demonstrated to trigger short-term or acute hyperalgesia but were now involved directly in allodynia, chronic hyperalgesia and neuropathic pain [33,34]. In this study, we found that the cerebellar FNS significantly alleviates the body weight loss (since day $21^{\text {st }}$ ) and sucrose preference (since day $17^{\text {th }}$ ), locomotor activities and rearing activities in open filed test (since Day $21^{\text {st }}$. Moreover, the stroke rats have a transient cytokines expression upregulation on day $7^{\text {th }}$ and $14^{\text {th }}$, comparing with sham rats. The PSD rats have significantly upregulated expression of cytokine since day $7^{\text {th }}$ to the day $21^{\text {st }}$. The PSD rats with FNS have significantly lower expression level of cytokines, which is consistence with the decrease of the depressive symptoms in the FNA rats.

The region salvaged by FNS corresponds to neurons which are estimated to be experiencing a postponed death process, not those dying neurotically and immediately resulting from the insult. For instance, the tissue salvaged by FNS after a focal ischemic injury aligns with a zone of the focal ischemic infarction peripheral to the ischemic core, known as the presumed ischemic penumbra [35]. Neurons in the ischemic core exhibit irreversible injury over a short period from excessive anoxic stress [36]. By contrast, the penumbra is hypermetabolic and has a partially preserved blood supply, which causes sublethal damage finally facilitating postponed apoptotic cell death [37, 38]. Overall, these findings increase the intriguing potential that FNS may not only inhibit inflammatory reactions, but also alleviate apoptotic processes resulting in neuronal death after an ischemic event [32]. In this study, we conducted the immunostaining of activated caspase- 3 and found that apoptosis is significantly higher in stroke group (Fig. 7B), and even higher in the PSD rats (Fig. 7C). The cerebellar FNS dramatically decreased the expression level of caspase-3 (Fig. 7D), suggesting that the FNS protects the cerebellar Purkinje cells from apoptosis induced by PSD.

Taken together, our study confirmed that Cerebellar FNS alleviates depressive-like behaviors in PSD rat model. Further mechanism study show that Cerebellar FNS protect Purkinje cells and decrease the expression level of cytokines in hippocampal tissue. In this study, we confirmed the dysfunction of cerebellar fastigial nucleus is involved in the pathogenesis of development of post-stroke depression; Secondly, poststroke depression experimental modelling could facilitate the development of novel therapeutic tool to treat post-stroke depression; Thirdly, such treatment may avoid the adverse effects caused by the medical treatment. This study shed the light to development of treatments to PSD patient in the future.

\section{Abbreviation}

FNS (fastigial nucleus electrical stimulation); PSD ( post-stroke depression); rCBF (regional cerebral blood flow); MCAO (Middle cerebral artery occlusion); OFT (open field test).

\section{Acknowledgement}

The study was supported by National Natural Science Foundation of China (Grant Nos. 81241050 and 81371461), Natural Science Foundation of Liaoning Province (Grant No. 2013022018), and Liaoning Medical College Youth Foundation (Grant No. Y2012Z014). 


\section{Cellular Physiology Cell Physiol Biochem 2017;41:1403-1412

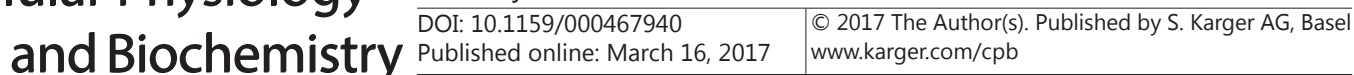 Zhang et al.: Cerebellar FNS Alleviates Depressive-Like Behaviors in PSD Rat Model}

\section{Disclosure Statement}

The authors declare that there is no conflict of interest

\section{References}

1 Robinson RG, Spalletta G: Poststroke depression: a review. Can J Psychiatry 2010;55:341-349.

$>2$ Hackett ML, Yapa C, Parag V, Anderson CS: Frequency of depression after stroke: a systematic review of observational studies. Stroke 2005;36:1330-1340.

-3 Kee NJ, Preston E, Wojtowicz JM: Enhanced neurogenesis after transient global ischemia in the dentate gyrus of the rat. Exp Brain Res 2001;136:313-320.

4 Lang UE, Beglinger C, Schweinfurth N, Walter M, Borgwardt S: Nutritional aspects of depression. Cell Physiol Biochem 2015;37:1029-1043.

5 Kronenberg G, Gertz K, Cheung G, Buffo A, Kettenmann H, Gotz M, Endres M: Modulation of fate determinants Olig2 and Pax6 in resident glia evokes spiking neuroblasts in a model of mild brain ischemia. Stroke 2010;41:2944-2949.

6 Buffo A, Vosko MR, Erturk D, Hamann GF, Jucker M, Rowitch D, Gotz M: Expression pattern of the transcription factor Olig2 in response to brain injuries: implications for neuronal repair. Proc Natl Acad Sci U S A 2005;102:18183-18188.

7 Wang SH, Zhang ZJ, Guo YJ, Sui YX, Sun Y: Involvement of serotonin neurotransmission in hippocampal neurogenesis and behavioral responses in a rat model of post-stroke depression. Pharmacol Biochem Behav 2010;95:129-137.

8 Wang SH, Zhang ZJ, Guo YJ, Teng GJ, Chen BA: Hippocampal neurogenesis and behavioural studies on adult ischemic rat response to chronic mild stress. Behav Brain Res 2008;189:9-16.

-9 Bugalho P, Correa B, Viana-Baptista M: [Role of the cerebellum in cognitive and behavioural control: scientific basis and investigation models]. Acta Med Port 2006;19:257-267.

10 Yun-Hong Y, Chih-Fan C, Chia-Wei C, Yen-Chung C: A study of the spatial protein organization of the postsynaptic density isolated from porcine cerebral cortex and cerebellum. Mol Cell Proteomics 2011;10:M110 007138.

11 Fu T, Stellmacher A, Znalesniak EB, Dieterich DC, Kalbacher H, Hoffmann W: Tff3 is expressed in neurons and microglial cells. Cell Physiol Biochem 2014;34:1912-1919.

12 Sui R, Zhang L, Min L, Yuan J, Li X: Cerebellar dysfunction may play an important role in post-stroke depression. Med Hypotheses 2009;72:643-646.

13 Su CY, Wuang YP, Lin YH, Su JH: The role of processing speed in post-stroke cognitive dysfunction. Arch Clin Neuropsychol 2015;30:148-160.

-14 Zhou P, Qian L, Zhou T, Iadecola C: Mitochondria are involved in the neurogenic neuroprotection conferred by stimulation of cerebellar fastigial nucleus. J Neurochem 2005;95:221-229.

15 Reis DJ, Berger SB, Underwood MD, Khayata M: Electrical stimulation of cerebellar fastigial nucleus reduces ischemic infarction elicited by middle cerebral artery occlusion in rat. J Cereb Blood Flow Metab 1991;11:810-818.

-16 Reis DJ, Kobylarz K, Yamamoto S, Golanov EV: Brief electrical stimulation of cerebellar fastigial nucleus conditions long-lasting salvage from focal cerebral ischemia: conditioned central neurogenic neuroprotection. Brain Res 1998;780:161-165.

17 Glickstein SB, Golanov EV, Reis DJ: Intrinsic neurons of fastigial nucleus mediate neurogenic neuroprotection against excitotoxic and ischemic neuronal injury in rat. J Neurosci 1999;19:4142-4154.

18 Lucassen PJ, Heine VM, Muller MB, van der Beek EM, Wiegant VM, De Kloet ER, Joels M, Fuchs E, Swaab DF, Czeh B: Stress, depression and hippocampal apoptosis. CNS Neurol Disord Drug Targets 2006;5:531-546.

19 Czeh B, Michaelis T, Watanabe T, Frahm J, de Biurrun G, van Kampen M, Bartolomucci A, Fuchs E: Stressinduced changes in cerebral metabolites, hippocampal volume, and cell proliferation are prevented by antidepressant treatment with tianeptine. Proc Natl Acad Sci U S A 2001;98:12796-12801.

-20 Zhao Z, Huang G, Wang B, Zhong Y: Inhibition of NF-kappaB activation by Pyrrolidine dithiocarbamate partially attenuates hippocampal MMP-9 activation and improves cognitive deficits in streptozotocininduced diabetic rats. Behav Brain Res 2013;238:44-47. 


\section{Cellular Physiology Cell Physiol Biochem 2017;41:1403-1412

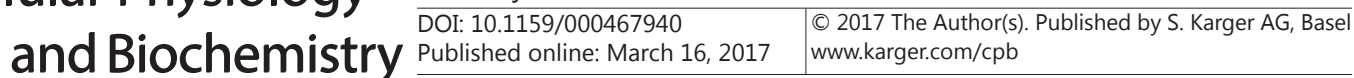 \\ Zhang et al.: Cerebellar FNS Alleviates Depressive-Like Behaviors in PSD Rat Model}

21 Gould E, Tanapat P: Stress and hippocampal neurogenesis. Biol Psychiatry 1999;46:1472-1479.

22 Willie CK, Colino FL, Bailey DM, Tzeng YC, Binsted G, Jones LW, Haykowsky MJ, Bellapart J, Ogoh S, Smith KJ, Smirl JD, Day TA, Lucas SJ, Eller LK, Ainslie PN: Utility of transcranial Doppler ultrasound for the integrative assessment of cerebrovascular function. J Neurosci Methods 2011;196:221-237.

23 Mraovitch S, Calando Y, Pinard E, Pearce WJ, Seylaz J: Differential cerebrovascular and metabolic responses in specific neural systems elicited from the centromedian-parafascicular complex. Neuroscience 1992;49:451-466.

24 Malonek D, Grinvald A: Interactions between electrical activity and cortical microcirculation revealed by imaging spectroscopy: implications for functional brain mapping. Science 1996;272:551-554.

-25 Hachinski V: Post-stroke depression, not to be underestimated. Lancet 1999;353:1728.

-26 Whyte EM, Mulsant BH, Vanderbilt J, Dodge HH, Ganguli M: Depression after stroke: a prospective epidemiological study. J Am Geriatr Soc 2004;52:774-778.

27 Gainotti G, Antonucci G, Marra C, Paolucci S: Relation between depression after stroke, antidepressant therapy, and functional recovery. J Neurol Neurosurg Psychiatry 2001;71:258-261.

28 Williams LS, Ghose SS, Swindle RW: Depression and other mental health diagnoses increase mortality risk after ischemic stroke. Am J Psychiatry 2004;161:1090-1095.

29 Pohjasvaara T, Vataja R, Leppavuori A, Kaste M, Erkinjuntti T: Depression is an independent predictor of poor long-term functional outcome post-stroke. Eur J Neurol 2001;8:315-319.

-30 Chemerinski E, Robinson RG, Kosier JT: Improved recovery in activities of daily living associated with remission of poststroke depression. Stroke 2001;32:113-117.

-31 Linden M, Baumann K, Rotter M, Schippan B: Posttraumatic embitterment disorder in comparison to other mental disorders. Psychother Psychosom 2008;77:50-56.

-32 Galea E, Glickstein SB, Feinstein DL, Golanov EV, Reis DJ: Stimulation of cerebellar fastigial nucleus inhibits interleukin-1beta-induced cerebrovascular inflammation. Am J Physiol 1998;275:H2053-2063.

33 Argall KG, Armati PJ, Pollard JD, Watson E, Bonner J: Interactions between CD4+ T-cells and rat Schwann cells in vitro. 1. Antigen presentation by Lewis rat Schwann cells to P2-specific CD4+ T-cell lines. J Neuroimmunol 1992;40:1-18.

-34 Arruda JL, Colburn RW, Rickman AJ, Rutkowski MD, DeLeo JA: Increase of interleukin-6 mRNA in the spinal cord following peripheral nerve injury in the rat: potential role of IL-6 in neuropathic pain. Brain Res Mol Brain Res 1998;62:228-235.

-35 Berger SB, Ballon D, Graham M, Underwood MD, Khayata M, Leggiero RD, Koutcher JA, Reis DJ: Magnetic resonance imaging demonstrates that electric stimulation of cerebellar fastigial nucleus reduces cerebral infarction in rats. Stroke 1990;21:III172-176.

-36 DeGirolami U, Crowell RM, Marcoux FW: Selective necrosis and total necrosis in focal cerebral ischemia. Neuropathologic observations on experimental middle cerebral artery occlusion in the macaque monkey. J Neuropathol Exp Neurol 1984;43:57-71.

-37 Choi DW: Ischemia-induced neuronal apoptosis. Curr Opin Neurobiol 1996;6:667-672.

-38 Guegan C, Sola B: Early and sequential recruitment of apoptotic effectors after focal permanent ischemia in mice. Brain Res 2000;856:93-100. 